\title{
Psicooncología
}

ISSN: 1696-7240

\section{Programa educativo sobre muerte y cuidados paliativos en los profesio- nales de la salud}

\author{
Leticia Ascencio Huertas ${ }^{1,2}$
}

Recibido: EL 6 de marzo de 2019 / Aceptado el 20 de marzo de 2019

Resumen: Introducción: La relación existente entre el personal de salud y la muerte, tienen una inevitable implicación emocional, ya que se vive y convive cotidianamente con la muerte. Objetivo: Conocer el efecto de un Programa Educativo sobre Muerte y Cuidados Paliativos (PEMyCP), fundamentado a partir de la noción de enfermedad y muerte en México. Método: Estudio cuasiexperimental comparativo, muestreo no probabilístico por conveniencia, diseño pretest-postest de un solo grupo, con profesionales de la salud en México. Los instrumentos utilizados fueron: Escala de actitudes ante los cuidados paliativos, Cuestionario de competencias profesionales y Escala de ansiedad ante la muerte de Templer. A través de análisis de frecuencias, pruebas t para muestras relacionadas y análisis de varianza ANOVA. Resultados: El PEMyCP tuvo un impacto positivo en las actitudes ante los cuidados paliativos $(\mathrm{t}=-2,0050, \mathrm{p}=0,44)$, en las capacidades profesionales $(\mathrm{t}=6.969, \mathrm{p}<0.0001)$, y ansiedad ante la muerte $(\mathrm{t}=2,991, \mathrm{p}=0,15)$. Se realizó un ANOVA, encontraron cambios significativos en conocimientos $(\mathrm{F}=8,5988, \mathrm{p}<0,0001)$ y habilidades $(\mathrm{F}=6,1197, \mathrm{p}<0,0001)$; sin embargo, no se identificaron cambios en actitudes $(\mathrm{F}=0,76131, \mathrm{p}=0,63738)$. En la ansiedad ante la muerte, se aprecian diferencias estadísticamente significativas $(\mathrm{F}=4,0048, \mathrm{p}=0,00919)$. Conclusiones: $\mathrm{El}$ PEMyCP tuvo un impacto favorable en conocimientos, habilidades y ansiedad ante la muerte, no siendo así en las actitudes ante la muerte y los cuidados paliativos, ya que se requiere de mayor tiempo para lograr modificarlas debido a que se adquieren no solo aspectos cognitivos, sino también afectivos y conductuales a través de la propia experiencia.

Palabras clave: programa educativo, muerte, cuidados paliativos, competencias profesionales, ansiedad ante la muerte.

\section{[en] Educational program on death and palliative care in health professionals}

\begin{abstract}
Introduction: The existing relationship between health personnel and death, have an inevitable emotional involvement, since they live and coexist daily with death. OBJECTIVE: To know the effect of an Educational Program on Death and Palliative Care (PEMyCP), based on the notion of illness and death in Mexico. Method: Comparative quasi-experimental study, non-probabilistic sampling for convenience, pretest-posttest design of a single group, with health professionals in Mexico. The instruments used were: Scale of attitudes before the palliative care, Questionnaire of professional competences and Scale of anxiety before the death of Templer. Through frequency analysis, tests $t$ for related samples and analysis of variance ANOVA. Results: The PEMyCP had a positive impact on the attitudes towards palliative care $(\mathrm{t}=-2.0050, \mathrm{p}=0.44)$, on professional abilities $(\mathrm{t}=6.969, \mathrm{p}=$

1 Servicio de Cuidados Paliativos, Instituto Nacional de Cancerología. Servicio de Cuidados Paliativos. México.

2 Doctorado en Ciencias Sociales y Administrativas, Universidad Chapultepec.

* Dirección de correspondencia: Leticia Ascencio Huertas. Instituto Nacional de Cancerología, Servicio de Cuidados Paliativos. Avenida San Fernando 22, Belisario Domínguez Sección XVI, Tlalpan, Ciudad de México, 14080, México. E-mail: lascencioh@incan.edu.mx; leash71@yahoo.com.mx
\end{abstract}


$0.00)$, and anxiety about death ( $\mathrm{t}=2.991, \mathrm{p}=0.15)$. ANOVA was performed, found significant changes in knowledge $(F=8.5988, p=0.0000)$ and abilities (skills $(F=6.1197, p=0.0000)$; however, no changes in attitudes were identified $(\mathrm{F}=0.76131, \mathrm{p}=0.63738)$. In the anxiety before death, are observed statistically significant differences $(\mathrm{F}=4.0048, \mathrm{p}=0.00919)$. Conclusions: The PEMyCP had a favorable impact on knowledge, abilities and anxiety in the face of death, but not in the attitudes towards death and palliative care, since it takes more time to modify them because not only cognitive aspects are acquired, but also affective and behavioral through the own experience.

Keywords: educational program, death, palliative care, professional abilities, anxiety about death.

Sumario. 1. Introducción 2. Método 3. Resultados 4. Conclusiones 5. Discusión 6. Consideraciones éticas 7. Referencias bibliográficas.

Cómo citar: Ascencio Huertas, L. Programa educativo sobre muerte y cuidados paliativos en los profesionales de la salud. Psicooncología 2019; 16(1):177-189. doi: 10.5209/PSIC.63654.

\section{Introduccion}

El miedo que tenemos a enfrentarnos con la muerte es un miedo ante lo desconocido, es una experiencia que nadie en vida podrá conocer en su totalidad, lo que nos lleva a generar altos niveles de ansiedad. Sin embargo, el ser consciente de nuestra finitud, promueve el desarrollo humano, logra la madurez psicoemocional y motiva a una vida plena.

De acuerdo con la Organización Panamericana de la Salud( ${ }^{(1)}$, y al informe sobre las enfermedades crónicas y promoción de la salud emitido por la Organización Mundial de la $\mathrm{Salud}^{(2)}$, las enfermedades crónicas son la causa principal de la muerte en todos los países de América. El 60\% de todas las defunciones se deben a enfermedades crónicas, las cuales han surgido como un problema y desafío para la salud pública, debido a sus efectos en la calidad de vida en los individuos afectados, ya que es causa de muertes prematuras y tiene efectos económicos importantes y subestimados en las familias, las comunidades y sociedad en general; entre las que destacan las cardiopatías, accidentes cerebrovasculares, el cáncer, las enfermedades respiratorias crónicas y la diabetes.

Los profesionales de la salud que se encargan de la atención a pacientes terminales pueden influir con intervenciones apropiadas en 2 procesos: en el proceso de morir y en el manejo del duelo de los familiares y el duelo propio ${ }^{(3)}$.

Sin embargo, no sólo el personal médico sufre el proceso del morir, sino todos los profesionales de la salud involucrados en la atención clínica, que en ocasiones viven la muerte de un paciente como una pérdida o un fracaso; algunos de los factores que determinan esta significación con la muerte suele asociarse con el temor, la intensidad del dolor, la relación que tienen con cada paciente, la estancia en el servicio, la causa de muerte y de sus propias experiencias con la muerte de familiares o amigos (historias de vida).

Algunos estudios han informado de la estrecha relación que existe entre el personal de salud y la muerte, con su inevitable implicación emocional ya que no sólo vive y convive cotidianamente con la muerte, sino que también afronta con el trabajo diario su propia muerte. Por lo que se requiere de mayor capacitación para abordar temas de relevancia al final de la vida ${ }^{(4)}$; conocimientos para afrontar las situaciones 
de cuidado ${ }^{(5)}$, fomentar la esperanza en los pacientes a través de reflexionar sobre sus valores, creencias y experiencia ${ }^{(6)}$, considerar la diversidad cultural, costumbres y rituales ${ }^{(7)}$ y percibir los condicionamientos sociales al momento de atender el proceso de muerte de los pacientes ${ }^{(8)}$.

Es importante mencionar que la literatura apunta a que en la educación para el cuidado al final de la vida en las profesiones de la salud, se precisa la preparación para atender a las personas que están muriendo y a sus seres queridos ${ }^{(9)}$. Diversos autores se han planteado, introducir el tema de la muerte en la enseñanza a través de áreas curriculares específicas, como ${ }^{(10)}$ quien define a la Pedagogía de la muerte como ámbito principal de la Pedagogía existencial, proponiendo algunas actividades didácticas para introducir el tema de la muerte en la escuela, desde áreas curriculares como lengua y literatura, ciencias naturales, música, filosofía, ciencias sociales o religión $^{(11)}$. Desde el año 1997 Gracia, proponía una Pedagogía laica de la muerte en el área de lengua y literatura a través del uso de simbolismos entre la psique (mente) y la muerte ${ }^{(12)}$ que aportan una serie de recursos y actividades para normalizar el tema de la muerte en la educación básica a través de cuentos y el dibujo como expresión simbólica para elaborar recursos frente a la ansiedad ante la muerte

En el 2003, De la Herrán y Mar Cortina, desarrollaron una serie de propuestas en torno a una educación para la muerte desde un enfoque complejo-evolucionista, fundamentado en la conciencia del valor de la muerte como objeto didáctico para la evolución y complejidad del conocimiento ${ }^{(13)}$. Años más tarde, Hegedus, Zana y Szabó ${ }^{(14)}$ proponen que los trabajadores de la salud y estudiantes de medicina y otras áreas de la salud, deben cubrir los mismos temas relacionados con la muerte, el morir y los aspectos psicosociales de los pacientes terminales.

Al respecto, Jones y Finlay ${ }^{(15)}$ informaron que aun cuando los estudiantes de Medicina pueden tener conocimientos médicos, todavía no existe el apoyo emocional, ni están suficientemente apoyados en el momento de la muerte de un paciente; y adaptarse a las características y necesidades propias de cada persona y población y que se incluya por su valor formativo en la educación de personas ${ }^{(16)(17)}$.

Algunos estudios realizados con médicos en formación de posgrado(18-20) encontraron que los propios médicos manifestaron no recibir atención ideal con respecto al manejo de la muerte de sus pacientes y que estaban interesados en formar parte de un equipo orientado a mejorar la atención al final de la vida.

Pero no olvidemos que otras disciplinas del área de la salud intervienen en la atención del paciente terminal, referente a la educación con respecto a la muerte en el área de enfermería hace referencia a la necesidad de incluir en los planes de estudio de pregrado, posgrado o curso de capacitación en enfermería el apoyo y cuidado físico y emocional al final de la vida ${ }^{(21-27)}$ Ya que de acuerdo con Gillan, Parmenter, van der Riet y Jeong ${ }^{(28)}$ y Gillan, Pamela, van der Riet, y Jeong ${ }^{(29)}$, la mayoría de los estudiantes de pregrado de enfermería están poco preparados para proporcionar los cuidados a pacientes al final de la vida y brindar atención a sus familias.

Gala León et al. ${ }^{(30)}$ establecen que una formación educativa adecuada para el personal de salud debería fomentar unas actitudes adecuadas hacia el enfermo terminal:1) Tomar consciencia de sus necesidades fisiológicas, sobre todo en lo referente al alivio del dolor físico y psíquico, 2) Desarrollar habilidades y actitudes de observación y escucha para poder identificar adecuadamente sus necesidades específicas, 3) Atender adecuadamente las necesidades informativas de los familiares y allegados, 4) Atender y ser sensible a las necesidades espirituales y religiosas del paciente, 5) Facilitar y 
disponer las mejores condiciones posibles del entorno, que hagan más cómodo y acogedor el habitáculo del enfermo, tanto para él como para sus familiares.

Por lo que podemos considerar que diversas especialidades del área de la salud comparten un objetivo principal: proporcionar a sus pacientes una buena muerte, descrita como "la que está libre de malestar y sufrimiento evitables a pacientes, familiares y cuidadores, de acuerdo con sus deseos y con los estándares clínicos, culturales y éticos"(31). Lo que hace necesario desarrollar programas educativos para el personal de salud, que fortalezcan el proceso de enseñanza y aprendizaje. Uno de los Modelos Educativos que ha mostrado eficiencia en el manejo de pacientes terminales, está basado en competencias, a través de conocimientos, habilidades y actitudes que se promueven en el personal de salud.

El Modelo educativo basado en competencias puede ayudar a proporcionar un contenido relevante clínicamente y estandarizado para los cuidados paliativos y los programas de educación. Además, las normas de competencia establecidas pueden ayudar a los profesionales de salud y para evaluar su propia formación e identificar los requisitos de educación ${ }^{(32)}$.

Bajo este panorama es importante que, en las instituciones de formación y desarrollo profesional en el área de la salud, se promueva el tema de la muerte con el objetivo de sensibilizarse ante el morir y el proceso de muerte de los pacientes terminales y su propio proceso de muerte. Considerando no solo la generación de conocimientos y habilidades sino involucrar los aspectos socioculturales, sus experiencias personales y profesionales con respecto a la muerte.

Por lo que el objetivo de la investigación fue evaluar el impacto que tienen un Programa Educativo sobre muerte y Cuidados Paliativos basado en competencias, con el fin de desarrollar conocimientos, habilidades y actitudes competentes en el personal de salud con respecto a la muerte y los cuidados paliativos desde una perspectiva interdisciplinar.

\section{Método}

Estudio prospectivo, cuasi experimental, transversal, con un diseño pretest-postest de un solo grupo.

\section{Participantes}

Fueron 66 profesionales de la salud que prestan sus servicios profesionales en diversas instituciones de salud, en diferentes regiones de México.

\section{Muestreo}

No probabilístico, no aleatorio y por conveniencia y que decidieron participar voluntariamente en el estudio

\section{Instrumentos}

- Escala de Actitudes ante los Cuidados Paliativos (Palliative Care Attitude Scale). Este cuestionario consta de 26 preguntas cortas y respuestas de opción múltiple relacionadas con la percepción del encuestado sobre el nivel existente de conocimiento, actitudes, creencias, y de experiencias sobre los cuidados paliativos y un reactivo cualitativo inicial que aborda el concepto de cuidados paliativos ${ }^{(33)}$. En su versión al español se obtuvo un Alpha de Cronbach de 0,807 ${ }^{(34)}$. 
Tabla 1. Características sociodemográficas, académicas y laborales

\begin{tabular}{|c|c|c|c|c|}
\hline \multicolumn{2}{|c|}{ Características } & \multicolumn{3}{|c|}{ Frecuencia / Porcentaje } \\
\hline \multirow[t]{2}{*}{ Genero } & Femenino & \multicolumn{3}{|c|}{$55 / 83,3$} \\
\hline & Masculino & \multicolumn{3}{|c|}{$11 / 16,7$} \\
\hline \multirow[t]{4}{*}{ Estado civil } & Solter@ & \multicolumn{3}{|c|}{$47 / 71,21$} \\
\hline & Casad@ & \multicolumn{3}{|c|}{$15 / 22,73$} \\
\hline & Divorciad@ & \multicolumn{3}{|c|}{$1 / 1,52$} \\
\hline & Unión Libre & \multicolumn{3}{|c|}{$3 / 4,55$} \\
\hline \multirow{3}{*}{$\begin{array}{l}\text { Entidades } \\
\text { Federativas }\end{array}$} & $\begin{array}{l}\text { Estados (Oaxaca y } \\
\text { Campeche) }\end{array}$ & \multicolumn{3}{|c|}{$26 / 39,4$} \\
\hline & Campeche) & & & \\
\hline & (Ciudad de México) & \multicolumn{3}{|c|}{$40 / 60,6$} \\
\hline \multirow[t]{4}{*}{ Profesión } & Psicología & \multicolumn{3}{|c|}{$35 / 53,03$} \\
\hline & Medicina & \multicolumn{3}{|c|}{$22 / 33,33$} \\
\hline & Enfermería & \multicolumn{3}{|c|}{$4 / 6,06$} \\
\hline & Trabajo Social & \multicolumn{3}{|c|}{$5 / 7,58$} \\
\hline \multirow[t]{10}{*}{ Grado de estudios } & Licenciatura & \multicolumn{3}{|c|}{$54 / 81,82$} \\
\hline & Maestría & & $7 / 10,61$ & \\
\hline & Especialidad & & $1 / 1,52$ & \\
\hline & Subespecialidad & & $4 / 6,06$ & \\
\hline & Estudiantes & & $3 / 4,5$ & \\
\hline & Servicio Social & & $7 / 10,61$ & \\
\hline & Trabajadores de base & & $43 / 65,15$ & \\
\hline & Médicos residentes & & $5 / 7,58$ & \\
\hline & Directivos & & $4 / 6,6$ & \\
\hline & Media & Máximo & Mínimo & Desv. Std. \\
\hline Edad & 27,6 & 66,0 & 20 & 8,6 \\
\hline Experiencia clínice & 3,8 & 20,0 & 0,0 & 8,4 \\
\hline
\end{tabular}

- Cuestionario de Evaluación de Competencias Profesionales. Auto aplicable de 20 reactivos, que fue adaptado para el presente estudio por los investigadores que determina la eficacia de las competencias técnicas o conocimientos, habilidades o destrezas y actitudinales, utilizando la escala likert de 4 rubros: 1) No competente, 2) Medianamente Competente, 3) Competente y 4) Altamente Competente. Con un Alpha de $0,923^{(35)}$.

- Escala de Ansiedad ante la muerte de Templer. Consta de 15 reactivos tipo Likert con valores asignados del 1 al 4 y que obtuvo una adecuada confiablidad con un alpha de 0,86 para población adulta ${ }^{(36)}$ y 0,83 para estudiantes $^{(37)}$.

- Programa Educativo sobre muerte y Cuidados Paliativos (PEMyCP). Consta de seis unidades de contenido: 1) La enfermedad terminal y la muerte en México, 2) criterios de comunicación con respecto a malas noticias (diagnóstico, pronóstico y muerte), 3) directrices de atención del paciente terminal y su familia, 4) aspectos bioéticos y legales en la atención del paciente terminal en México, 5) creencias, miedos y actitudes ante la muerte en el personal de salud y 6) rituales y ceremonias ante la muerte en México. 
Las actividades del programa se diseñaron a partir de técnicas vivenciales considerando objetivos, cultura y contexto de los participantes para facilitar la expresión de sentimientos, emociones y palabras; y promover la reflexión, el análisis, y el aprendizaje basado en la experiencia, para actuar con mayor competencia técnica e interpersonal.

\section{Resultados}

En el presente estudio se contó con la participación de 78 sujetos, de los cuales de acuerdo con los criterios de exclusión fueron eliminados 12 por lo que la muestra total fue de 66, de los cuales $55(83,3 \%)$ mujeres y $11(16,67 \%)$ varones.

En la Tabla 1, se observa que la mayoría de los sujetos son solteros con $47(71,2 \%)$ y 15 casados (22,7\%). Principalmente psicólogos $(53,0 \%)$ y médicos $(33,3 \%)$; a nivel licenciatura (81,8\%). La media en tiempo de años de experiencia en el área clínica fue de 3,8 años lo que nos habla de una población joven en la atención de pacientes. La media de edad fue de 27,6 años.

En la Escala de Actitudes ante los Cuidados Paliativos en el reactivo 1 de tipo cualitativo, (¿Qué significa para usted el término Cuidados Paliativos?), se encontró que el termino CP se asoció inicialmente a calidad de vida $(25,8 \%)$ a la atención integral en la última etapa de la vida $(16,7 \%)$ y al control del dolor y los cuidados al final de la vida $(10,6 \%)$ y que con la implementación del PEMyCP se conservó la asociación calidad de vida al paciente y su familia $(9,1 \%)$ pero se modificó el concepto de $\mathrm{CP}$ al considerar que se refiere a la atención al paciente sin opción a tratamiento curativo $(7,2 \%)$ y con la atención integral en la última etapa de la vida $(6,8 \%)$. Como se observa en la Figura 1

Figura 1. Porcentajes del reactivo 1. Escala de Actitudes ante los Cuidados Paliativos: ¿Qué significa para usted el término Cuidados Paliativos?: Pretest y postest

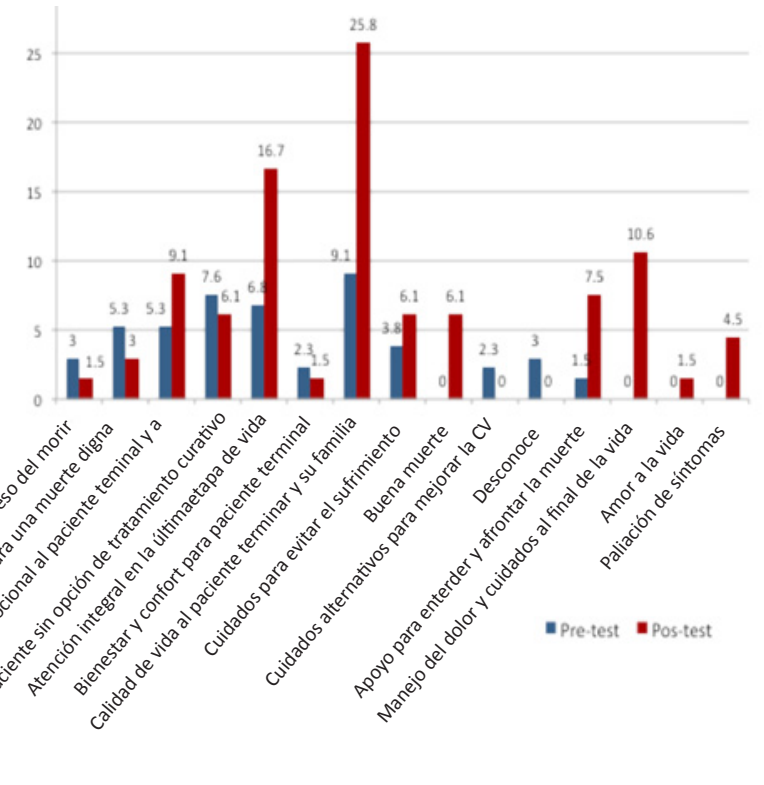


El PEMyCP modificó las actitudes ante los cuidados paliativos $\left(\mathrm{t}_{(65)}=-2,0050\right.$, $\mathrm{p}=0,44)$, las competencias profesionales $\left(\mathrm{t}_{(65)}=6,969, \mathrm{p}<0,0001\right)$, y la ansiedad ante la muerte $\left(\mathrm{t}_{(65)}=2,991, \mathrm{p}=0,15\right)$, como se muestra en la Tabla 2 .

Tabla 2. Estadísticas descriptivas y pruebas t para muestras relacionadas de cada Escala

\begin{tabular}{|c|c|c|c|c|c|c|c|c|}
\hline & Media & Des. Std & Mín. & Mediana & Máx. & $\mathbf{t}$ & gl & $\begin{array}{c}\text { Sig. } \\
\text { (bilateral) }\end{array}$ \\
\hline \multicolumn{9}{|c|}{ Escala de Actitudes ante los Cuidados Paliativos } \\
\hline Pre-test & 79,5 & 11,5 & 44,0 & 79,0 & 108,0 & \multirow{2}{*}{$-2,050$} & \multirow{2}{*}{65} & \multirow{2}{*}{0,44} \\
\hline Post-test & 82,5 & 12,3 & 44,0 & 84,0 & 104,0 & & & \\
\hline \multicolumn{9}{|c|}{ Cuestionario de Evaluación de Competencias Profesionales } \\
\hline Pre-test & 51,4 & 9,5 & 31,0 & 52,0 & 78,0 & \multirow{2}{*}{$-6,969$} & \multirow{2}{*}{65} & \multirow{2}{*}{0,00} \\
\hline Post-test & 59,6 & 8,3 & 43,0 & 59,5 & 78,0 & & & \\
\hline \multicolumn{9}{|c|}{ Escala de Ansiedad ante la muerte de Templer } \\
\hline Pre-test & 25,1 & 5,5 & 16,0 & 25,0 & 43,0 & \multirow{2}{*}{2,913} & \multirow{2}{*}{65} & \multirow{2}{*}{0,15} \\
\hline Post-test & 23,4 & 5,4 & 15,0 & 23,0 & 78,0 & & & \\
\hline
\end{tabular}

Con respecto al análisis de varianza (ANOVA), la Figura 2 muestra el Cuestionario de Evaluación de Competencias profesionales en la subescala de conocimientos, se obtuvieron diferencias estadísticamente significativas, $\mathrm{F}_{(5,126)}=8,5988, \mathrm{p}<0,001$.

Figura 2. ANOVA la subescala de conocimientos del Cuestionario de Evaluación de Competencias Profesionales: Pretest y postest

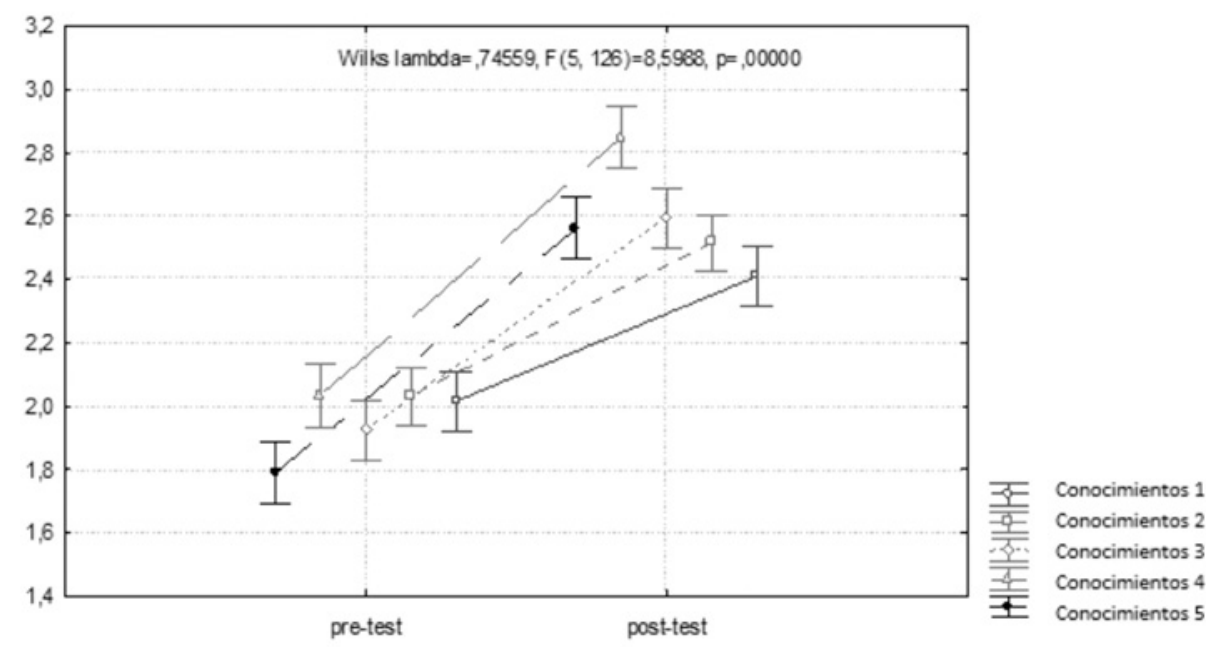


En la subescala de habilidades también se obtuvieron diferencias estadísticamente significativas $F_{(7,124)}=6,1197 p<0,001$ como lo observa en la Figura 3 y en la subescala de actitudes, no se encontraron diferencias estadísticamente significativas, $\mathrm{F}_{(8,123)}=0,76131, \mathrm{p}=0,63738$. Lo que nos lleva a considerar que las actitudes al ser adquiridas y aprendidas a lo largo del tiempo, requiere para su modificación no solo componentes cognitivos, sino también afectivos y conativos.

Figura 3. ANOVA de la subescala de habilidades del Cuestionario de Evaluación de Competencias Profesionales: Pretest y pos-test.

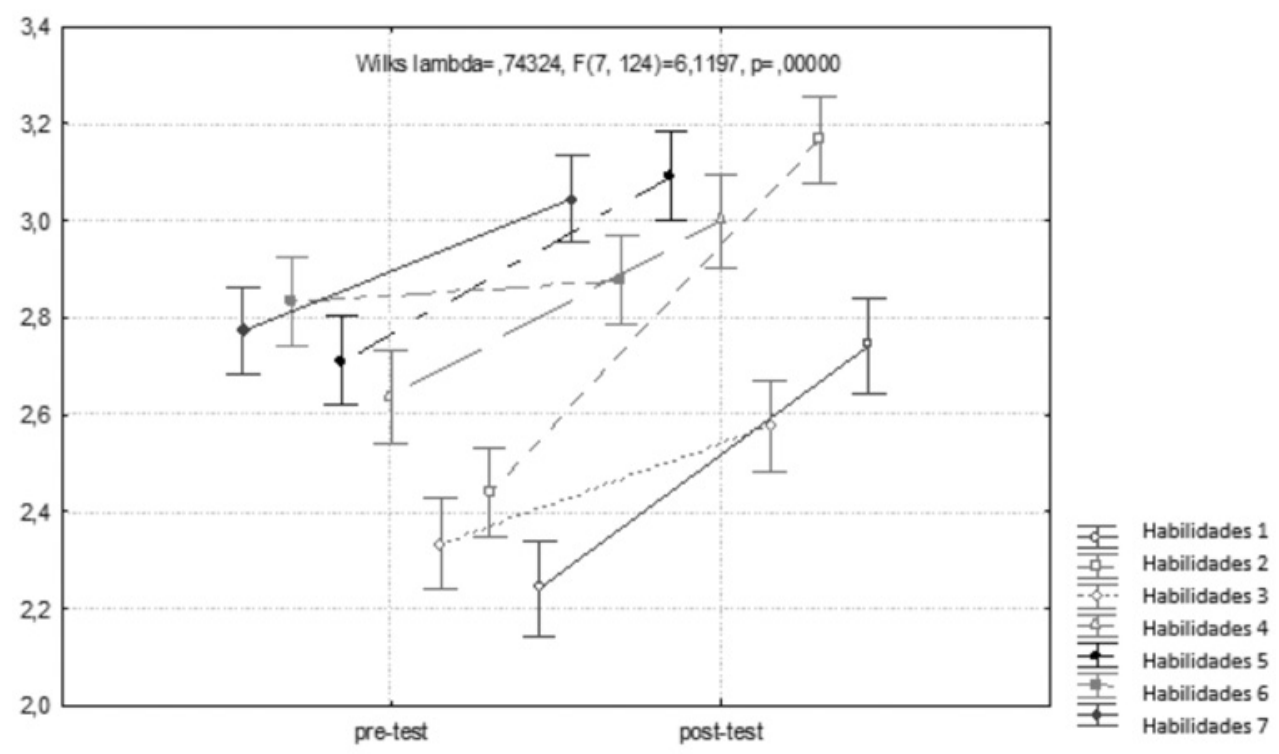

En el análisis de varianza (ANOVA) de las 3 subescalas de la Escala de Ansiedad ante la muerte de Templer, existen diferencias estadísticamente significativas, $\mathrm{F}_{(3,128)}$ $=4,0040, \mathrm{p}=0,05$ Enfatizando que al realizar el PEMyCP, el miedo a la muerte y a la agonía disminuyen, sin embargo el miedo a que la vida llegue a su fin se incrementa, probablemente porque los participantes no habían reflexionado acerca del tema de la muerte y el morir y menos con técnicas vivenciales (Ver Figura 4).

\section{Conclusiones}

Con base en los resultados obtenidos, podemos afirmar que los médicos han sido entrenados en reconocer y diagnosticar las enfermedades, pero desconocen todo lo que gira alrededor de la muerte y los cuidados paliativos y sería revelador que aprendieran a paliar síntomas físicos, emocionales y hasta espirituales y a brindar el acompañamiento hasta el final de la vida de sus pacientes. 
Figura 4. ANOVA de las 3 subescalas de la Escala de Ansiedad ante la Muerte de Templer:

Pre-test y pos-test

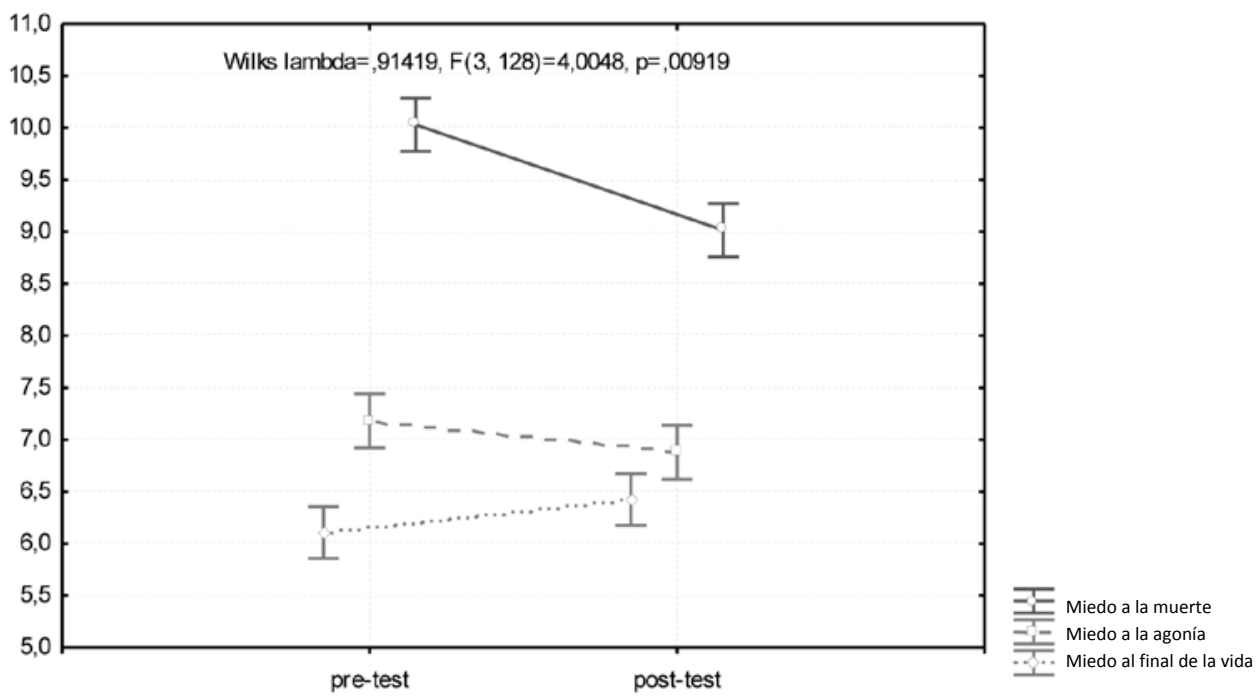

Es importante aludir a que el Programa Educativo sobre muerte y cuidados paliativos basado en competencias, se desarrolló en función de la noción de enfermedad y de muerte del mexicano, por lo cual los temas que se consideraron fueron: la enfermedad y la muerte en México, criterios de comunicación con respeto a las malas noticias, directrices de atención del paciente terminal y su familia, aspectos bioéticos y legales en la atención del paciente terminal en México, creencias, miedos y actitudes ante la muerte en el personal de salud y rituales y ceremonias ante la muerte en México.

Dentro de los resultados más relevantes encontramos que los profesionales de la salud están de acuerdo en la importancia que tienen actualmente los cuidados paliativos en México pero que se requiere de una preparación específica para brindar estos cuidados al final de la vida desde todas las disciplinas para el manejo integral del paciente terminal y su familia ya que es limitada la preparación durante la formación profesional para abordar temas como: la comunicación de malas noticias, la atención emocional, a la familia como parte de la atención paliativa, los aspectos espirituales y los socioculturales.

Con relación a las actitudes frente a los cuidados paliativos se destaca que no solo se debe involucrar el control del dolor sino considerar otros síntomas físicos que deben ser manejados y controlados en la medida de lo posible, e involucrar a la familia en la toma de decisiones y considerar los tratamientos sintomáticos y aspectos emocionales, socioculturales y espirituales del paciente y su familia, así como reconocer que la muerte de pacientes genera emociones en los profesionales de la salud, que deberían manejarse y contar con el apoyo de profesionales de la salud especialistas en Psicología.

Con respecto a las competencias adquiridas con la implementación del Programa Educativo sobre muerte y Cuidados Paliativos, podemos concluir que contar con la suficiente información y la ejecución de actividades que sensibilicen al personal de salud en el manejo de la muerte y los cuidados paliativos se fortalecen los conocimientos y habilidades necesarias para brindar una adecuada atención 
a pacientes al final de la vida, sin embargo cabe destacar que para modificar las actitudes ante la muerte y el morir, se requiere de mayor tiempo, ya que se van adquiriendo con la experiencia, por lo que se sugiere introducir estos temas desde el de pregrado de cada una de las disciplinas involucradas en la atención del paciente terminal e introducir el manejo de la muerte a través de talleres vivenciales, grupos de experiencia o seminarios permanentes de muerte, para sensibilizar de forma anticipada los profesionales de la salud.

\section{Discusión}

La construcción de programas basados en competencias es un gran reto en el sistema educativo como la establece Díaz Barriga ${ }^{(38)}$ y más en el campo de la salud, ya que además de centrarse en los procesos cognitivos y en el comportamiento se deben considerar diversos factores como la cultura, los valores, la cosmovisión, las etnias, las creencias y las actitudes. Sin embargo se complica aún más la implementación de estos programas al relacionarse con un diagnóstico terminal, la agonía y la muerte, ya que aun cuando son temas esenciales en el área de la salud son inexistentes, motivo por el cual se propone a través de la presente investigación, promover la atención de las personas al final de la vida de forma integral con una buena atención a través de adecuadas habilidades interpersonales, conocimientos clínicos, respeto por las personas, los valores, las actitudes y la experiencia personal y profesional; tal como lo establece Breslin $^{(39)}$ al mencionar que la cultura es la cosmovisión y los valores de un grupo.

En el contexto mexicano se complica aún más, por la gran diversidad cultural que existe, sin embargo, al mismo tiempo se enriquece por el gran valor y aporte de las diferentes concepciones ante la vida y la muerte en las diferentes regiones de México y la gran riqueza cultural desde la época prehispánica hasta nuestros días.

Un punto relevante en la formación de los profesionales de la salud en el manejo del paciente terminal, es el proceso de comunicación, ya que afecta de manera significativa en la relación médico-paciente, en el impacto emocional, en la adherencia terapéutica y en la toma de decisiones; sin embargo no se le ha dado importancia adecuada y son pocos los recursos educativos que promueven estrategias de comunicación como un factor evidente en la prestación de servicios de salud, como lo reveló Sanz Ortiz ${ }^{(40)}$ al referirse a que uno de los déficits en la carrera de medicina es ausencia de entrenamiento en el área de comunicación, a pesar de que son justo los médicos, los responsables de comunicar los diagnósticos, las posibilidades de tratamiento y hasta el pronóstico de vida, por lo que se requiere incorporar estas habilidades para que los médicos aprendan a comunicarse de forma eficiente y efectiva, haciendo énfasis en que saber comunicar no solo implica trasmitir información sino saber escuchar e intercambiar emociones, considerar las creencias, miedos y temores, del paciente al médico pero también del médico al paciente, ya que es un asunto que involucra no solo a un profesional de la salud con su paciente, sino a un humano frente a otro. Ante lo cual, es necesario establecer nuevas pautas para desarrollar en el personal de salud habilidades de comunicación y empatía en una dinámica de interacción humana, ya que comunicar es un arte.

El manejo de la muerte y los cuidados paliativos son un área de oportunidad para los profesionales de la salud en los diferentes niveles de atención de salud en México para mejorar la atención de las personas al final de la vida. Y evitar la negación de la 
muerte, que se encuentra asociada a la falta de preparación profesional en cuidados paliativos y la muerte, el poco entrenamiento en estrategias de afrontamiento personal, y la estructura organizativa de los hospitales, por lo que una de las propuestas más importantes de esta investigación en promover en el personal de salud las competencias funcionales, habilidades y actitudes profesionales para la atención del paciente terminal a través de los cuidados paliativos y el manejo de la muerte, tomando en cuenta los aspectos multidimensionales, el contexto cultural de cada persona, familia y población, ya que está ligada a un cambio de valores sociales, que se trasluce en los comportamientos, como lo refieren Chocarro González, González Fernández, Salvadores y Venturini ${ }^{(41)}$. Por lo cual es importante abordar los temas de muerte y los cuidados paliativos desde la familia, en los contextos cotidianos, sociales, económicos, culturares y educativos y no solo durante la formación profesional o ante la experiencia del final de la vida vida.

Para futuras investigaciones se sugiere incrementar el tamaño de la muestra y considerar la diversidad cultural, así como las diversas especialidades en el campo de la salud.

\section{Consideraciones éticas}

El estudio es de riesgo mínimo, debido a que durante la aplicación de los instrumentos se pudo haber generado alguna molestia, sin embargo, en todo momento se explicó a los participantes que en caso necesario contaría con el apoyo psicológico sin costo alguno.

Cada participante conto con el consentimiento informado y se explicó el objetivo y riesgo de la presente investigación Y se llevó a cabo de acuerdo con la Declaración de Helsinki, a las Buenas Prácticas Clínicas y a la normativa de la Ley General de Salud de México.

\section{Referencias bibliográficas}

1. Organización Panamericana de la Salud (PAHO) (2007), Estrategia Regional y Plan de Acción para un Enfoque Integrado Sobre la Prevención y el Control de las Enfermedades Crónicas. [Internet]. [Acceso el 27 de febrero de 2019]. Disponible en: http://www1. paho.org/Spanish/AD/DPC/NC/reg-strat-cncds.pdf

2. OMS | Prevención de las enfermedades crónicas [Internet]. WHO. [Acceso el 27 de febrero de 2019]. Disponible en: http://www.who.int/chp/chronic_disease_report/ part1/es/

3. Olivé, L. La muerte algunos problemas filosóficos. Ciencia UNAM, 1995; 38, 30-35. [Internet]. [Acceso el 27 de febrero de 2019]. Disponible en: http://revistas.unam.mx/ index.php/cns/article/viewFile/11449/10774

4. Trueman I, Parker J. Exploring community nurses' perceptions of life review in palliative care. J Clin Nurs 2006;15:197-207.

5. Vargas-Danza E, Pacheco-Rodríguez A, Arellano-Martínez M, Martínez-González L y Galicia-Rodríguez L. Percepción de enfermería en torno a la muerte de pacientes en etapa terminal. Rev Enferm Instituto Mexicano Seguro Social, 2008; 16(1), 37-44. [Internet]. [Acceso el 27 de febrero de 2019]. Disponible en: http://revistaenfermeria.imss.gob.mx/ editorial/index.php/revista_enfermeria/article/view/515/588 
6. Mok E, Lau K, Lam W, Chan L, Ng J, Chan K. Health-care professionals' perspective on hope in the palliative care setting. J Palliat Med. 1 de julio de 2010;13:877-83.

7. Sneesby L, Satchell R, Good P, Riet P van der. Death and dying in Australia: perceptions of a Sudanese community. J Adv Nurs 2011;67:2696-702.

8. Perdigon AGC, Strasser G. El proceso de muerte y la enfermería: un enfoque relacional. Reflexiones teóricas en torno a la atención frente a la muerte. Physis Rev Saúde Coletiva 2015;25:485-500.

9. Todaro-Franceschi V, Spellmann M. End of life care pedagogy, death attitudes, and knowing participation in change. J Nurs Educ Pract 2013; 3, 120-125. [Internet]. ResearchGate. doi: 10.5430/jnep.v3n2p120

10. Mèlich J.C. El concepto existencial de hombre y la educación permanente. Rev Esp Pedagog 1989;47:137-44.

11. Rodriguez-Herrero P, De la Herrán A y Cortina Selva M. Antecedentes de pedagogía de la muerte en España. Enseñañnza y Teaching 2012; 30: 175-95.

12. Portero PF, Porto ABP. Muerte y educación. Tarbiya Rev Investig E Innov Educ [Internet]. 15 de febrero de 2017 [citado 27 de febrero de 2019];0(33). Disponible en: https://revistas. uam.es/tarbiya/article/view/7255

13. Diaz JA, Cortina M, Herán H de la. Pedagogía de la muerte a través del cine. Madrid: Universitas 2011. Teor Educ:3.

14. Hegedus K, Zana A, Szabó G. Effect of end of life education on medical students' and health care workers' death attitude. Palliat Med 2008;22:264-9.

15. Jones R, Finlay F. Medical students' experiences and perception of support following the death of a patient in the UK, and while overseas during their elective period. Postgrad Med J 2014;90:69-74.

16. Rodríguez Herrero P. Educación para la muerte y el duelo: hacia la formación integral de las personas con discapacidad intelectual. X Conferencia Internacional de Ciencias de la Educación, Camagüey (Cuba). Citado en: Rodríguez Herrero, P., Herrán, A. de la y Cortina M. Antecedentes de Pedagogía de la Muerte en España. Enseñanza \& Teaching. Revista Interuniversitaria de Didáctica 2012; 30: 175-95.

17. Rodríguez-Herrero P, Torre G de la. Educación para la muerte y el duelo en personas con discapacidad intelectual. [Acceso el 27 de febrero de 2019]; Disponible en: https:// www.academia.edu/26082565/EDUCACI\%C3\%93N_PARA_LA_MUERTE_Y_EL_ DUELO_EN_PERSONAS_CON_DISCAPACIDAD_INTELECTUAL

18. Rappaport W, Prevel C, Witzke D, Fulginiti J, Ballard J, Wachtel T. Education about death and dying during surgical residency. Am J Surg 1991;161:690-2.

19. DiMaggio JR. Educating psychiatry residents about death and dying: A national survey. Gen Hosp Psychiatry 1993;15:166-70.

20. Bergman J, Lorenz KA, Acquah-Asare S, Scales CD, Ryan G, Saigal CS, et al. Urologist attitudes toward end-of-life care. Urology 2013;82:48-52.

21. Lyons GJ. Bereavement and death education - a survey of nurses' views. Nurse Educ Today 1988;8:168-72.

22. Tomás Sábado J, Guix Llistuella E. Ansiedad ante la muerte: efectos de un curso de formación en enfermeras y auxiliares de enfermería. Enferm Clínica. 2001;11:104-9.

23. Aradilla-Herrero A, Tomás-Sábado J. Efectos de un programa de educación emocional sobre la ansiedad ante la muerte en estudiantes de enfermería. Enferm Clínica 2006; 16:321-6.

24. Schlairet MC. End-of-life nursing care: statewide survey of nurses' education needs and effects of education. J Prof Nurs. 2009; 25:170-7. doi: 10.1016/j.profnurs.2008.10.005. 
25. Pascual MA. Análisis de los niveles de ansiedad ante la muerte de los profesionales de enfermería de cuidados críticos | NURE Investigación 2011:1-12. [citado 27 de febrero de 2019]; Disponible en: http://www.nureinvestigacion.es/OJS/index.php/nure/article/view/521

26. Kent B, Anderson NE, Owens RG. Nurses' early experiences with patient death: the results of an on-line survey of Registered Nurses in New Zealand. Int J Nurs Stud 2012;49:1255-65.

27. Todaro-Franceschi V, Spellmann M. End of life care pedagogy, death attitudes, and knowing participation in change. J Nurs Educ Pract 2012;3:120.

28. Gillan PC, Parmenter G, van der Riet PJ, Jeong S. The experience of end of life care simulation at a rural Australian University. Nurse Educ Today 2013;33:1435-9.

29. Gillan PC, van der Riet PJ, Jeong S. End of life care education, past and present: a review of the literature. Nurse Educ Today 2014;34:331-42.

30. Gala León FJ, Lupiani Jiménez M, Raja Hernández R, Guillén Gestoso C, González Infante J M, Villaverde Gutiérrez MC et al. Actitudes psicológicas ante la muerte y el duelo: Una revisión conceptual. Cuadernos de Medicina Forense. 2002;30: 39-50 [Internet]. [Acceso el 4 de marzo de 2019]. Disponible en: https://www.researchgate. net/publication/244952534_Actitudes_psicologicas_ante_la_muerte_y_el_duelo_Una_ revision_conceptual

31. Selecky PA, Eliasson CAH, Hall RI, Schneider RF, Varkey B, McCaffree DR, et al. Palliative and end-of-life care for patients with cardiopulmonary diseases: American College of Chest Physicians position statement. Chest 2005;128:3599-610.

32. Becker R. The development of core competencies for palliative care educators. Int $\mathrm{J}$ Palliat Nurs 2007;13:377-83.

33. Kain V, Gardner G, Yates P. Neonatal palliative care attitude scale: development of an instrument to measure the barriers to and facilitators of palliative care in neonatal nursing. Pediatrics 2009; 123:e207-213.

34. Ascencio L. Adaptación en español de la escala de actitudes ante cuidados paliativos: confiabilidad y análisis factorial. Psicooncología; 2015; 12: 367-81. doi. 10.5209/rev_ PSIC.2015.v12.n2-3.51015

35. Ascencio L. La muerte desde una perspectiva interdisciplinar: implementación de un programa educativo en cuidados paliativos. Tesis Doctoral Inedita. Universidad Chapultepec, México; 2014.

36. Tomás-Sábado J, Gómez-Benito J. Psychometric properties of the Spanish form of Templer's Death Anxiety Scale. Psychol Rep 2002;91(3 Pt 2):1116-20.

37. Rivera-LedesmaAy Montero-LópezLM, Propiedades psicométricas de la escala de ansiedad ante la muerte de Templer en sujetos mexicanos. Diversitas: Perspectivas en Psicología 2010; 6: 135-40. [Internet]. ResearchGate. [Acceso el 4 de marzo de 2019]. Disponible en: https://www.researchgate.net/publication/ 283393492_Propiedades_psicometricas_de_la_ escala_de_ansiedad_ante_la_muerte_de_Templer_en_sujetos_mexicanos

38. Díaz Barriga A. Construcción de programas de estudio en la perspectiva del enfoque de desarrollo de competencias. Perfiles Educativos XXXVI, 2014; 142-62.Construcción de programas de estudio en la perspectiva del enfoque de desarrollo de competencias [Internet]. [Acceso el 4 de marzo de 2019]. Disponible en: http://www.scielo.org.mx/ scielo.php?script=sci_arttext\&pid=S0185-26982014000100009

39. Brislin RW. Understanding culture's influence on behavior. Harcourt College Publishers; 2000. p. 468

40. Sanz Ortiz J. Final de la vida: ¿puede ser confortable? Med Clínica 2001;116:186-90.

41. Chocarro González L, González Fernández R, Salvadores Fuentes P, Venturini Medina C. Negación de la muerte y su repercusión en los cuidados. Med Paliat 2012;19:148-54. 
